\title{
Noch immer nicht angekommen? - Strukturelle Geschlechterungleichheit im Deutschen Bundestag
}

\author{
Ina E. Bieber iD
}

Eingegangen: 9. November 2020 / Überarbeitet: 10. Oktober 2021 / Angenommen: 11. Oktober 2021 / Online publiziert: 7. Dezember 2021

(C) Der/die Autor(en) 2021

Zusammenfassung Trotz formaler Gleichstellung sind Frauen im Deutschen Bundestag immer noch unterrepräsentiert. Forschungsergebnisse legen nahe, dass institutionelle Faktoren entscheidend zur strukturellen Diskriminierung von Frauen in der Politik beitragen. Vor allem Mehrheitswahlsysteme und im rechten Parteispektrum verortete Parteien führen weltweit und auch in Deutschland zu niedrigen Frauenanteilen in den Parlamenten. Vor dem Hintergrund der anstehenden Wahlrechtsreform des Bundestags - deren Auftrag es auch ist, eine ,[...] gleichberechtigte Repräsen$\operatorname{tanz}$ von Frauen und Männern auf den Kandidatenlisten und im Bundestag zu erreichen“ - untersucht der Artikel, welche strukturellen Faktoren die Wahlchancen von Frauen bei Bundestagswahlen beeinflusst haben. Besonderes Augenmerk wird dabei auf die Auswirkungen des Wahlsystems und der ideologischen Ausrichtung der Parteien gelegt. Der verwendete Datensatz umfasst Kandidatinnen und Kandidaten bei Bundestagswahlen von 1953-2017. Damit ermöglicht er Analysen auf Individualebene, die auch die Untersuchung der Nominierungsqualität berücksichtigen, also der individuellen Erfolgschancen einer Kandidatur. Die Ergebnisse des Papiers zeigen, dass der Mechanismus des Mehrheitswahlrechts, aber auch die Qualität der Nominierung und die Parteizugehörigkeit die Wahlchancen von Frauen beeinflussen und somit Stellschrauben sind, um den Frauenanteil im Bundestag langfristig zu erhöhen.

Schlüsselwörter Deskriptive Repräsentation · Frauen · Kandidaturen · Wahlsystem · Parteien · Diskriminierung

Erklärung zur Datenverfügbarkeit Die Autorin hat die Daten selbst erhoben und aufbereitet. Es ist geplant, die Daten zeitnah zu veröffentlichen.

Ina E. Bieber $(\bowtie)$

Gesellschaftswissenschaften, Goethe-Universität Frankfurt am Main, Theodor-W. Adorno-Platz 6, Hauspostfach 15, 60323 Frankfurt am Main, Deutschland

E-Mail: bieber@soz.uni-frankfurt.de 


\section{Still not There?-Structural Gender Inequality in the German Bundestag}

Abstract Despite formal equality, women are still underrepresented in the German Bundestag. Research findings suggest that institutional factors contribute decisively to structural discrimination against women in politics. Majority voting systems and right-wing parties, in particular, lead to low proportions of women in legislatures worldwide as well as in Germany. In light of the upcoming electoral reform of the Bundestag-whose mandate is also to achieve an "equal representation of women and men on candidate lists and in the Bundestag" - the study examines which structural factors have influenced women's electoral chances in elections of the Bundestag. Special focus is put on the effects of the electoral system and the ideological party orientation. The data set used includes candidates in federal elections from 1953 to 2017 and thus allows individual-level analyses that also take into account the study of nomination quality-i.e., the individual chances of success of a candidacy. The results of the paper show that not only the mechanism of majority voting but also the quality of the nomination and the party affiliation of the candidates influence the electoral chances of women and thus can be factors for increasing the share of women in the Bundestag in the long term.

Keywords Descriptive representation - Women · Candidacy · Election system · Parties · Discrimination

\section{Ausgangslage und Anliegen}

„Deutschland auf Weltniveau“ titelte die taz am 23. November 2005, einen Tag nach der Wahl Angela Merkels zur ersten Bundeskanzlerin Deutschlands und stellte fest, dass mit ihrer Wahl nun weltweit zehn Frauen als Regierungschefinnen an der Macht sind (die tageszeitung 2005). Auch 2021 werden in nur 12 Ländern die Regierungsgeschäfte von einer Frau geführt (UN Women 2021). Nimmt man dies als Maßstab, so war Deutschland mit Angela Merkel viele Jahre auf „Weltniveau“. Ein Blick auf die Frauenanteile im Bundestag, in den Landesparlamenten und in den Kommunen macht jedoch schnell deutlich, dass in der Legislativen mengenmäßig keinesfalls von ,Weltniveau“ zu sprechen ist. Im Bundestag ist der Frauenanteil 2017 erstmals wieder auf 31,2\% gesunken (siehe Abb. 1), in den Landesparlamenten liegt er durchschnittlich bei 29,9\% und in den Kommunalparlamenten beläuft er sich auf 27,7\% (BMBF 2020). Darüber hinaus werden derzeit nur zwei von 16 Bundesländern von einer Frau geführt $(12,5 \%)$.

Die Forderung nach deskriptiver Repräsentation, also der äquivalenten Vertretung von Bevölkerungsgruppen in den Parlamenten, ist normativ wünschenswert, aber in der Umsetzung höchst problematisch. Das Parlament als repräsentativer Spiegel der Gesellschaft würde bedeuten, dass nicht nur Frauen, sondern auch andere Bevölkerungsgruppen wie Arbeiter*innen, Arbeitslose, Jugendliche oder Menschen mit Migrationshintergrund proportional zur Bevölkerung vertreten sein müssen (Abels et al. 2018; Brunsbach 2011; Davidson-Schmich 2016). Dies wird in Arbeitsparla- 


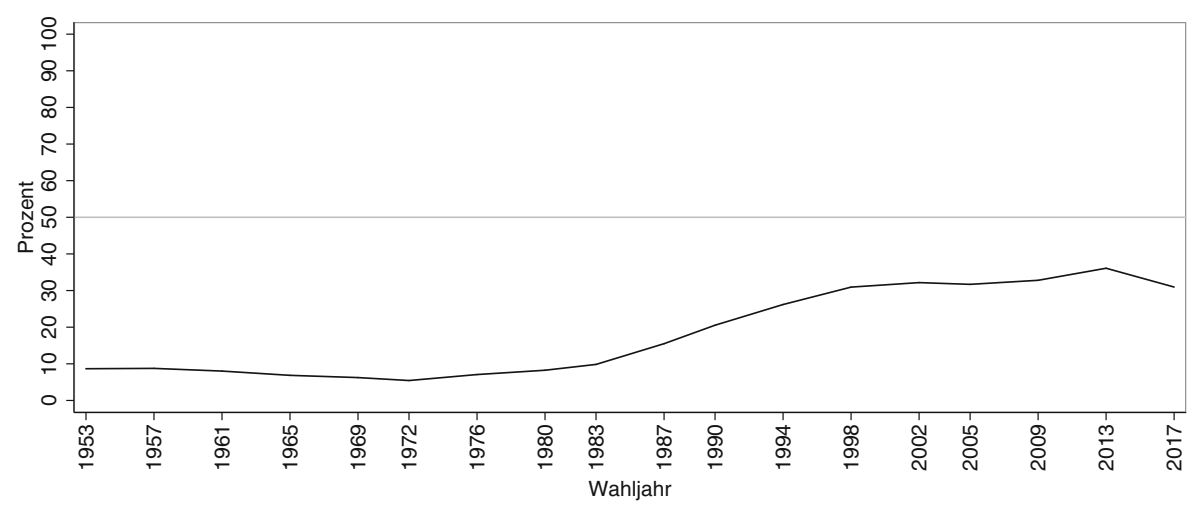

Abb. 1 Frauenanteil im Bundestag im Zeitverlauf (in \%)

menten wie in Deutschland nur selten erreicht, und es ist fraglich, ob der politische Output von Parlamenten tatsächlich stärker von den Gruppenmerkmalen der Abgeordneten als von der Parteipolitik determiniert wird (Brunsbach 2011). Unabhängig davon ist es zweifelhaft, ob in einem gleichberechtigten Staat denjenigen, die sich beteiligen wollen, durch institutionelle Mechanismen systematisch geringere Repräsentationschancen eingeräumt werden sollten. Dies gilt nicht zuletzt mit Blick auf Art. 3 GG, der die Gleichheit aller Menschen vor dem Gesetz fordert (Bundestag 2021).

Die aktuelle Forschung zeigt weitgehend, dass strukturelle Faktoren, die durch staatliche Maßnahmen verändert werden könnten, die Chancen von Frauen auf eine Vertretung systematisch verringern können: So belegen nationale und internationale Vergleichsstudien, dass Frauen in Mehrheitswahlsystemen tendenziell geringere Repräsentationsquoten aufweisen als in Verhältniswahlsystemen (Kenworthy und Malami 1999; Moser 2001; Paxton et al. 2010; Rule 1987; Vengroff et al. 2003; Deutschland: Bieber 2013). Andere Studien kommen zu dem Ergebnis, dass Frauen in linken Parteien größere Chancen auf ein Mandat haben als in rechten Parteien (Caul Kittilson 2006; Deutschland: Bieber 2013).

Angesichts dieser außergewöhnlich guten Forschungslage ist zu klären, warum dieses Thema einer erneuten Betrachtung bedarf. Drei Gründe sprechen dafür: Zentral ist die anstehende Gesetzesreform und die damit verbundene günstige Gelegenheit, aktuelle Forschungsergebnisse in den politischen Prozess einzubringen. Das Wahlrecht für die Bundestagswahl, dessen Ausgestaltung auch in vielen Landesparlamenten Anwendung findet, ist derzeit in einem Reformprozess. Erste Schritte zur Verkleinerung des Bundestags wurden bereits beschlossen und umgesetzt. Umfassende Änderungen sind für die nächste Legislaturperiode nach der Bundestagswahl 2021 geplant. Die eingesetzte Reformkommission soll bis Mitte 2023 insbesondere Maßnahmen empfehlen, ,um eine gleichberechtigte Repräsentanz von Frauen und Männern auf den Kandidatenlisten und im Bundestag zu erreichen“ (Bundestag 2020). Dieser Auftrag ist eine Gelegenheit, um auch auf strukturelle Mechanismen hinzuweisen, die die Frauenanteile systematisch negativ beeinflussen und die bei 
der anstehenden Wahlrechtsreform entsprechend berücksichtigt und geändert werden könnten.

Zweitens bedürfen die bisherigen wissenschaftlichen Arbeiten einer Aktualisierung und Vertiefung. Zwar gibt es bereits einige Studien, die untersuchen, inwiefern sich das Wahlsystem und die Parteiideologien auf die Repräsentation von Frauen in der (deutschen) Politik auswirken. Doch während sich beispielsweise die Arbeit von Davidson-Schmich (2016) auf freiwillige Geschlechterquoten konzentriert und die Analyse von Fortin-Rittberger und Eder (2013) auf aggregierte Daten zurückgreift, endet die auf Individualdaten basierende Längsschnittstudie von Bieber (2013) mit der Bundestagswahl 2009, deren Zeitreihe nicht nur verlängert, sondern methodisch optimiert und vertieft werden kann.

Drittens machen es aktuelle Entwicklungen notwendig, sich mit diesem Thema auseinanderzusetzen. So beobachtet Bieber (2020) eine „doppelte Dynamisierung“ bei der Verteilung der Mandate in den deutschen Landtagen zwischen 2015 und 2020, die negative Folgen für die Frauenanteile in den Parlamenten hat: Die erste Dynamisierung ist auf den massiven Mandatsgewinn der AfD zurückzuführen. Da die Mehrheit der AfD-Kandidat*innen männlich ist, wurden die Parlamente dadurch systematisch männlicher. Bei der zweiten Dynamisierung handelt es sich um eine veränderte Aufteilung der Wahlkreismandate zwischen SPD und CDU/CSU. Durch die Schwächung der SPD gingen die Wahlkreismandate zwischen 2015 und 2020 häufig an die Unionsparteien, die im Wahlkreis überproportional häufig Männer nominiert hatten. Dies führte ebenso dazu, dass die Parlamente wieder männlicher wurden.

Diese drei Gründe sind der Anlass, die Mechanismen des deutschen Wahlsystems und der Parteienlandschaft in diesem Beitrag erneut dahingehend zu untersuchen, wie sie sich auf die Repräsentationschancen von Frauen und Männern auswirken. Dies geschieht auf Grundlage eines Datensatzes, der es ermöglicht, die Selektionsmechanismen von der Kandidatur bis zum Mandat auf der individuellen Ebene der Kandidatinnen und Kandidaten für die Bundestagswahl 1953-2017 zu beleuchten. Denn erst eine geschlechtsspezifische Analyse des Auswahlprozesses von einer Kandidatur zu einem Mandat kann feststellen, ob die weibliche Unterrepräsentation dadurch zu erklären ist, dass weniger Frauen kandidiert haben oder tatsächlich systematische Benachteiligung vorliegt und Frauen dadurch signifikant niedrigere Chancen auf Wahlerfolg haben. Darüber hinaus stellt sich die Frage, welche Rolle dabei die Art der Kandidatur, die Parteizugehörigkeit zum linken bzw. zum rechten Spektrum und die Erfolgsaussicht der jeweiligen Kandidatur spielen?

Das Papier untergliedert sich in zwei Abschnitte: Im ersten Teil werden die grundlegenden, theoretischen Mechanismen des Wahlsystems und der Parteiideologie kurz vorgestellt und die Auswirkungen dieser Mechanismen auf die weibliche Kandidatur und Repräsentation in einem Längsschnittvergleich für Deutschland deskriptiv dargestellt. Der Fokus liegt dabei auf der Repräsentation von Frauen nach Wahlsystemtyp und Parteiideologie. Damit werden die Ergebnisse der Arbeit von Bieber (2013) um die Bundestagswahljahre 2013 und 2017 erweitert. Die deskriptiven Beobachtungen aus dem ersten Teil werden in einem zweiten Teil anhand von vier Hypothesen für Deutschland multivariat getestet. Untersucht werden sowohl der 
langfristige Geschlechtereffekt von 1953-2017 als auch der querschnittliche bei der Bundestagswahl 2017.

\section{Theoretische und deskriptive Betrachtungen}

\subsection{Wahlsystem}

Internationale Vergleiche zeigen, dass die Repräsentationsraten von Frauen in Mehrheitswahlsystemen niedriger als in Verhältniswahlsystemen sind (Kenworthy und Malami 1999; Moser 2001; Paxton et al. 2010; Rule 1987; Vengroff et al. 2003). Dies wird auf die Konkurrenzsituation im Nominierungsprozess und die damit verbundenen (vermeintlich besseren) Wahlchancen von Männern zurückgeführt. Auch in Deutschland sind deutliche Unterschiede zwischen den Geschlechtern zwischen den Wahlsystemtypen zu beobachten, sowohl auf der Ebene der Abgeordneten als auch auf der Ebene der Kandidat*innen (vgl. Bieber 2013).

Dies wird in Abb. 2 deutlich, in der die Anteile von Frauen an Wahlkreis- und Listenmandaten im Zeitverlauf dargestellt sind. Die Linien der Wahlkreis- und Listenmandate verlaufen weitgehend parallel, aber auf unterschiedlichen Niveaus. Der Anteil an Listenmandaten, der von Frauen gewonnenen wurde, lag immer höher als der Anteil der gewonnenen Wahlkreismandate. Seit Anfang der 1980er-Jahre ist bei beiden Gruppen ein Anstieg des Frauenanteils zu beobachten, wobei der Anteil bei den Listenmandaten stets höher war als bei den Wahlkreismandaten und auch 2017 noch besteht. So wurde 2017 nur einer von fünf Wahlkreisen von einer Frau gewonnen $(21,4 \%)$, während fast vier von zehn Listenmandaten an Frauen gingen. Bei der Betrachtung dieses Unterschieds ist es wichtig, die Mechanismen der Verhältnis- und Mehrheitswahl zu berücksichtigen: Bis 2017 wurden Wahlkreismandate überwiegend von den damals ,großen“ Parteien (SPD, CDU/CSU, im Osten auch AfD und Linke) gewonnen, während Listenmandate in größerem Umfang an Grüne, Linke und AfD gingen, was einen Teil der Diskrepanz erklären kann.

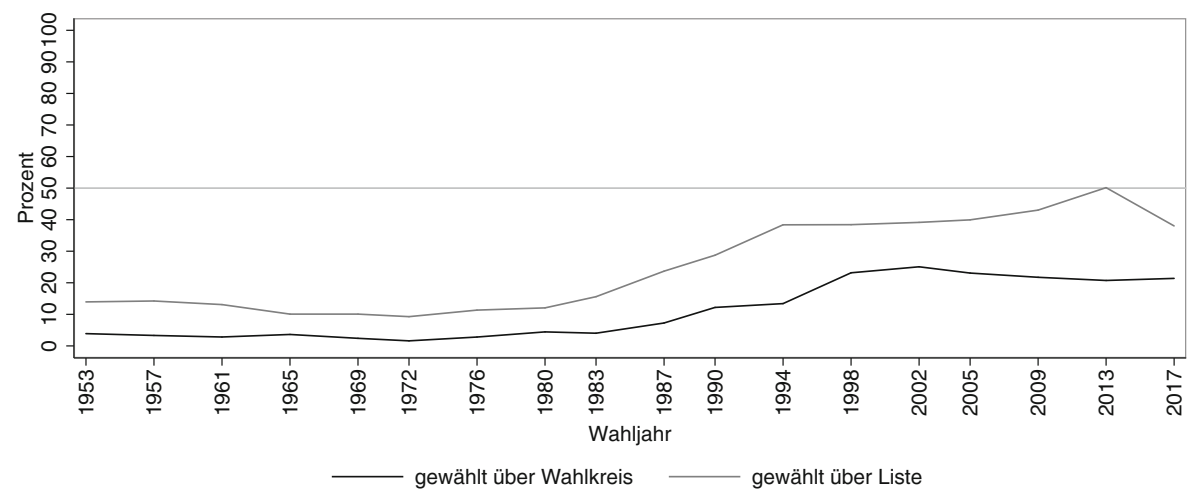

Abb. 2 Frauenanteil an Wahlkreis- und Listenmandaten im Zeitverlauf (in \%) 
Abb. 2 zeigt jedoch nur die letzte Stufe zur politischen Macht - die Stufe der gewählten Abgeordneten. Parteiarbeit ist aber eine „Ochsentour“ von ganz unten nach ganz oben (Hellmann 2020). Um Diskriminierungsprozesse adäquat zu beurteilen, lohnt es sich, einen Schritt zurückzugehen und bereits Kandidaturen zu betrachten (vgl. Fortin-Rittberger und Eder 2013; Paxton et al. 2010; Siaroff 2000; Vengroff et al. 2003).

Für den Wahlerfolg in Mehrheitswahlsystemen ist die Popularität der einzelnen Kandidat*innen innerhalb des Wahlkreises besonders wichtig, ebenso wie die Bereitschaft der Kandidat*innen, sich dem öffentlichen Wahlkampf zu stellen. Männer werden überdurchschnittlich häufig in Wahlkreisen nominiert (Norris 2004; Matland und Studlar 1996, S. 713). In Verhältniswahlsystemen haben Frauen dagegen bessere Chancen auf eine Nominierung. Die Parteien nutzen dabei das Nominierungsverfahren, um ihre bevorzugten Kandidat*innen ins Parlament zu bringen. Verhältniswahlsysteme erleichtern den Parteien auch die Umsetzung von Geschlechterquoten, da Frauen gezielt für aussichtsreiche Sitze nominiert werden können (Norris 2004).

Im Rahmen des personalisierten Verhältniswahlsystems in Deutschland können Personen bei Bundestagswahlen ausschließlich in Wahlkreisen, ausschließlich auf Parteilisten oder als sogenannte Doppelkandidat*innen im Wahlkreis sowie auf der Parteiliste antreten. Abb. 3 zeigt die Frauenanteile an diesen drei Arten von Kandidaturen für die Bundestagswahl in einer Längsschnittperspektive. Es ist sehr deutlich zu erkennen, dass der Anteil der Frauen, die nur in einem Wahlkreis kandidieren, auch heute noch sehr gering ist und seit 1987 bei rund 15\% liegt (2017: 15,9\%). Der Anteil der Frauen, die ausschließlich auf Listen kandidieren, war dagegen schon immer deutlich höher (2017: 43,7\%). Der stetige Anstieg der weiblichen Doppel-

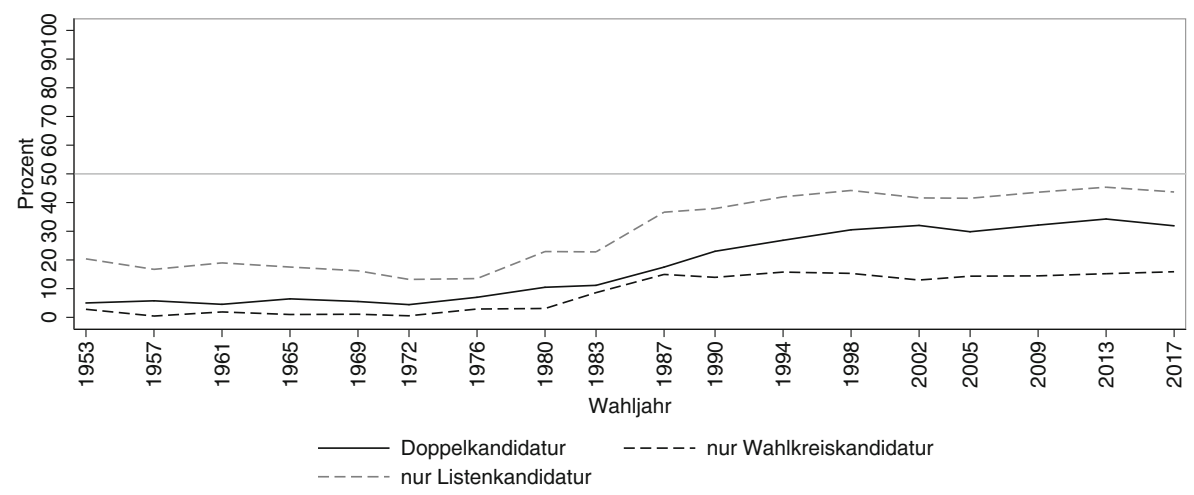

Abb. 3 Frauenanteil an Wahlkreis-, Listen- und Doppelkandidaturen im Zeitverlauf (in \%)

\footnotetext{
1 Das Verhältnis von Wahlkreis-, Listen- und Doppelkandidaturen hat sich über die Zeit verändert. Während bei der Wahl 1953 noch 43,1\% ausschließlich auf der Liste, 20,6\% ausschließlich im Wahlkreis und nur 36,4\% eine Doppelkandidatur innehatten, belief sich der Anteil an Doppelkandidat*innen bei der Wahl 2017 auf 50,7\% (ausschließlich Wahlkreiskandidat*innen: 20,0\%, ausschließlich Listenkandidaturen $29,3 \%$ ).
} 


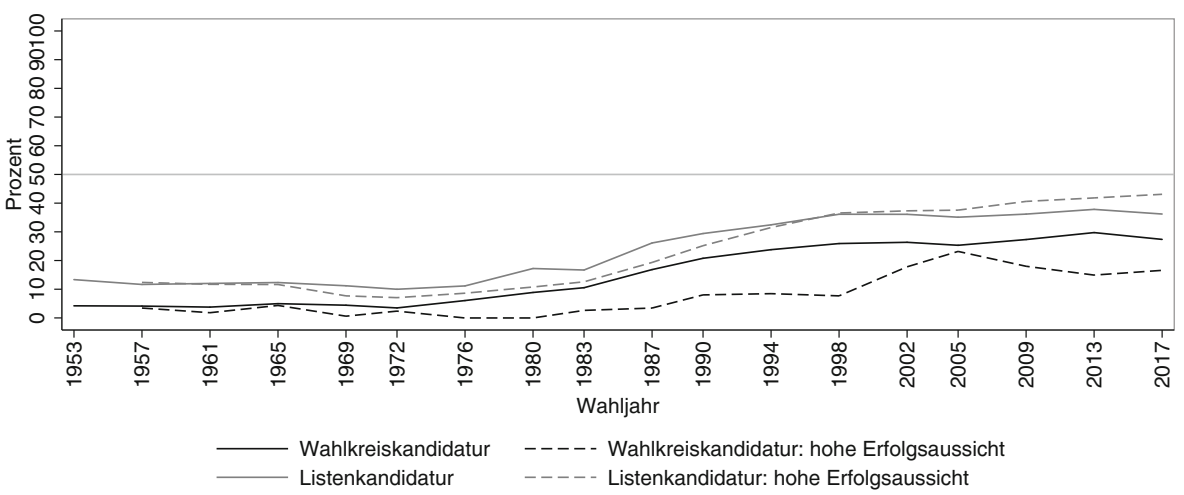

Abb. 4 Frauenanteil an Wahlkreis- und Listenkandidaturen im Zeitverlauf (in \%)

kandidaturen von 1972-2002, mit 31,9\% im Jahr 2017, ist auffallend und lohnt eine gesonderte Untersuchung. ${ }^{1}$

Noch wichtiger ist aber die Aussicht auf Erfolg - also die Frage, wie aussichtsreich der Wahlkreis ist, in dem eine (weibliche) Person kandidiert bzw. wie aussichtsreich die Platzierung auf einer Parteiliste ist (siehe Manow und Nistor 2009; Schmitt und Wüst 2004). Dieser Grund wird häufig zur Erklärung der niedrigen Frauenanteile herangezogen. In Analysen mit Aggregatdaten kann dieser Zusammenhang zwischen individueller Kandidatur, Erfolgsaussichten und tatsächlichem Wahlerfolg auf der individuellen Ebene jedoch nicht hergestellt werden, da die Daten nicht miteinander verknüpft sind. Zu diesem Zweck muss die individuelle Nominierungsqualität - im Folgenden auch ,,aussichtsreiche Kandidatur“ oder ,Erfolgsaussicht" genannt - jeder Kandidatur berechnet werden. Mit dem vorliegenden Datensatz ist dies möglich. ${ }^{2}$

Abb. 4 zeigt, dass der Anteil der Frauen in den Wahlkreisen mit hoher Erfolgsaussicht (schwarze, gestrichelte Linie) immer niedriger ist als der Frauenanteil an Wahlkreiskandidaturen insgesamt (schwarze, durchgehende Linie). Für 2017 bedeutet dies beispielsweise, dass der Frauenanteil in den Wahlkreisen insgesamt 27,4\% betrug, in den Wahlkreisen mit hoher Erfolgsaussicht aber nur 16,6\%. Bis 1990 ist ein vergleichbares Muster auch bei den Listenkandidaturen zu beobachten - allerdings auf einem höheren Niveau. Im Jahr 1998 wendet sich das Blatt und der Anteil der Frauen an aussichtsreichen Listenkandidaturen (graue, gestrichelte Linie) liegt seitdem über dem Frauenanteil an den Listenkandidaturen insgesamt (graue, durchgehende Linie), wenn auch nur geringfügig. Dies deutet darauf hin, dass Frauen bei Listenkandidaturen insgesamt höhere Erfolgsaussichten haben als Männer. Abb. 4 unterstreicht somit die geschlechtsspezifischen Unterschiede zwischen Listen- und Wahlkreiskandidaturen.

\footnotetext{
2 Details zur Berechnung der Nominierungsqualität siehe Abschn. 3.2.
} 


\subsection{Parteienlandschaft}

Parteien spielen eine entscheidende Rolle dabei, ob Frauen motiviert, nominiert und gewählt werden, weshalb ihnen auch die Rolle des „Gatekeepers“ zugeschrieben wird (Caul Kittilson 2006; Luhiste 2015). Studien haben gezeigt, dass Frauen sowohl seltener von Parteien nominiert werden als auch seltener von sich aus kandidieren wollen (Allik 2015; Bernstein 1986; Bieber 2013; Kenworthy und Malami 1999; Inglehart und Norris 2003; Preece et al. 2016; Schwindt-Bayer 2005). Neben einer Geschlechterquote ist die ideologische Ausrichtung einer Partei ein besonders relevanter, parteispezifischer Faktor zur Erklärung der strukturellen Unterrepräsentation von Frauen: Ausgehend von der Links-Rechts-Verortung der Parteien (vgl. hierzu Manifesto Project 2021; Scherer 2020) werden Kandidatinnen in Parteien des linken Spektrums größere Wahlchancen eingeräumt als solchen in Parteien des rechten Spektrums (vgl. Caul 2001; Erzeel und Caluwaerts 2015; Ondercin und Welch 2009; Stockemer und Sundström 2017).

Abb. 5 zeigt die Geschlechterunterschiede differenziert nach Verortung der Partei im Parteienspektrum und Erfolgsaussicht. ${ }^{3}$ Erstens ist zu erkennen, dass der Anteil der Kandidaturen von Frauen im linken Parteienspektrum insgesamt und bei aussichtsreichen Kandidaturen seit Ende der 1980er-Jahre deutlich höher ist als bei den Parteien des rechten Spektrums. Zweitens ist zu beobachten, dass bei den Kandidaturen des linken Spektrums der Anteil der Frauen an aussichtsreichen Kandidaturen seit 1994 höher ist als der Anteil an den Kandidaturen insgesamt. Dies deutet darauf hin, dass Frauen in links verorteten Parteien in der Nominierungsphase überdurchschnittlich viele aussichtsreiche Platzierungen erhalten. Besonders starke Effekte sind seit den 1980er-Jahren zu beobachten, was mit der Etablierung der Grünen und der Linken und der zunehmenden Einführung von Geschlechterquoten - zunächst vor allem in Parteien des linken Spektrums - zusammenzuhängen scheint. Demgegenüber waren die Frauenanteile im rechten Parteienspektrum insgesamt über

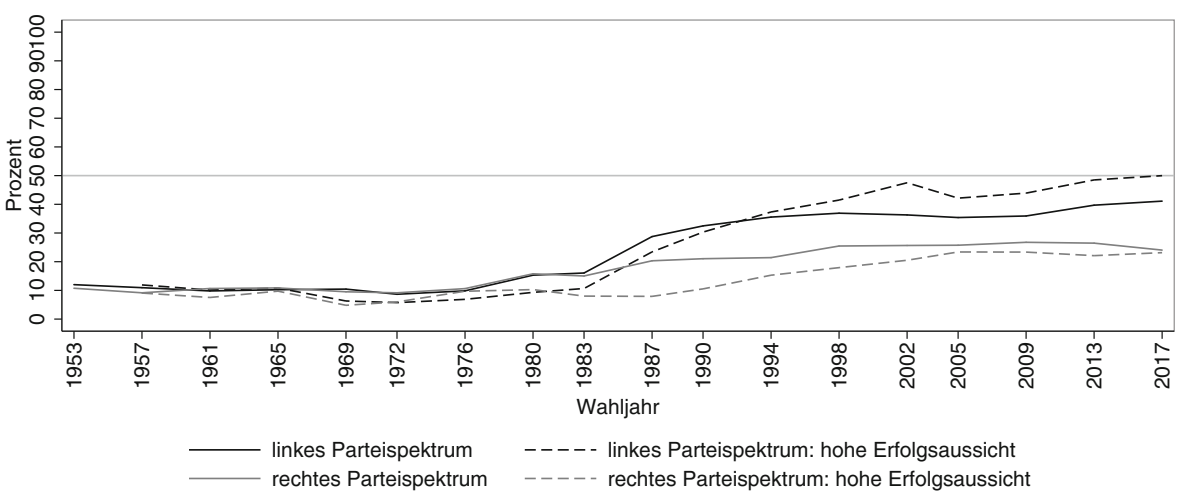

Abb. 5 Frauenanteil an Kandidaturen differenziert nach Parteispektrum und Erfolgsaussicht (in \%)

\footnotetext{
3 Die Linke, die Grünen und die SPD wurden dem linken Parteispektrum zugeordnet, die FDP, die CDU, die CSU und die AfD dem rechten Parteispektrum.
} 
den gesamten Zeitraum höher als die Frauenanteile in rechten Parteien mit hoher Erfolgsaussicht. Dies legt nahe, dass Frauen in rechten Parteien auf schlechteren Plätzen kandidieren als ihre männlichen Kollegen.

\section{Multivariate Analysen}

Die bisherigen Betrachtungen haben gezeigt, dass der Frauenanteil im Deutschen Bundestag im Zeitverlauf gestiegen ist, aber seit Anfang der 1990er-Jahre auf einem Niveau von rund einem Drittel verharrt. Darüber hinaus lassen sich deutliche Unterschiede in deskriptiver Hinsicht bei den Frauenanteilen an Wahlkreis- und Listenmandaten sowie entlang des Parteienspektrums feststellen. Diese Ergebnisse allein lassen jedoch noch kein abschließendes Urteil darüber zu, ob Frauen bei Bundestagswahlen durch das Wahlsystem und die Parteiideologie strukturell benachteiligt werden. Erst multivariate Analysen, bei denen der individuelle Wahlerfolg in Bezug zur Kandidatur gesetzt wird und weitere Einflussfaktoren kontrolliert werden, ermöglichen es, gegebenenfalls geschlechterdiskriminierende Selektionsmechanismen nachzuweisen.

\subsection{Hypothesen}

Ein zentrales Ergebnis der bisherigen deskriptiven Analyse ist, dass Frauen seit Bestehen der Bundesrepublik eine geringere Repräsentationsquote haben. Strukturelle Diskriminierung läge aber nur dann vor, wenn die Wahlchancen von Frauen signifikant geringer wären als die von Männern, was mit Hypothese 1 untersucht werden soll:

H1 Die Wahlchancen von Frauen sind geringer als die von Männern.

Die deskriptiven Ergebnisse zeigen auch, dass bezüglich der Chancen je nach Wahlsystem und Parteiideologie geschlechtsspezifische Unterschiede festzustellen sind. Der Anteil der Frauen an Listenkandidaturen war im Vergleich zu Wahlkreisoder Doppelkandidaturen am höchsten. Sind aber auch geschlechtsspezifische Wahlchancen in Bezug auf die Art der Kandidatur zu beobachten, die auf eine strukturelle Diskriminierung hinweisen? Hypothese 2 lautet daher:

H2 Die Wahlchancen von Frauen sind bei reinen Listenkandidaturen höher als bei reinen Wahlkreiskandidaturen. Die Wahlchancen sind bei Doppelkandidaturen am höchsten.

Weiterhin konnte deskriptiv gezeigt werden, dass Frauen in Parteien des linken Spektrums häufiger vertreten sind als in Parteien des rechten Spektrums. Aber lässt sich dies nur deskriptiv beobachten oder lassen sich diese besseren Wahlchancen auch bei einer multivariaten Analyse nachweisen? Hypothese 3 lautet: 
H3 Die Wahlchancen von Frauen sind in Parteien des linken Spektrums höher als in Parteien des rechten Spektrums.

Auch wenn deskriptiv nachgewiesen werden kann, dass Listenkandidaturen und die Zugehörigkeit zu einer linken Partei zu einer höheren Nominierungsqualität führen, ist diese Qualität für Frauen im Gesamten geringer als die für Männer. Deshalb ist es interessant zu betrachten, ob sich dieser Zusammenhang auch bei einer multivariaten Analyse zeigt. Hypothese 4 lautet daher:

H4 Die Nominierungsqualität von Frauen ist schlechter als die von Männern.

\subsection{Daten und Methoden}

Der verwendete Datensatz umfasst Bundestagskandidat*innen von 1953-2017. ${ }^{4}$ Es wurden Kandidat*innen aller erfolgreichen Parteien berücksichtigt. ${ }^{5}$ Diese Vollerhebung beinhaltet sozialstrukturelle und politische Informationen von insgesamt 32.436 Kandidat*innen. ${ }^{6}$ Aufgrund des gemischten Wahlsystems mit Mehrheitsund Verhältniselementen bietet der deutsche Fall ein besonderes analytisches Potenzial, um die geschlechtsspezifische Wirkungsweise des Wahlsystems zu betrachten. Wie bereits erwähnt, werden Individualdaten der Kandidat*innen verwendet, was das Potenzial für Analysen zusätzlich erhöht. ${ }^{7}$

Alle Berechnungen beruhen auf Regressionen. Es werden sowohl Analysen zum tatsächlichen Wahlerfolg als auch Analysen zur Nominierungsqualität durchgeführt. Die abhängigen Variablen sind demnach der Wahlerfolg (1: ,gewählt“, 0: ,nicht gewählt") und die Nominierungsqualität (1: ,aussichtsreich“, 0: ,,nicht aussichtsreich“ bzw. im Falle der Listenqualität eine kontinuierliche Variable von 0-1). ${ }^{8}$

4 Die Autorin hat die Daten selbst erhoben und aufbereitet.

5 Ab 1953 werden Kandidat*innen von SPD, CDU/CSU und FDP einbezogen. Kandidat*innen der Grünen, der Die Linken und der AfD werden ab 1983 (Grüne), 1990 (Die Linke) bzw. 2017 (AfD) berücksichtigt. Obwohl die FDP im Jahr 2013 keine Mandate gewinnen konnte, sind die Kandidat*innen für dieses Jahr in den Datensatz und in die Berechnungen einbezogen.

6 Der Anteil der Kandidatinnen beträgt 22,8\% (25.029 Männer; 7407 Frauen) und der Anteil der Amtsträgerinnen 19,6\% (8097 Männer; 1969 Frauen).

7 Viele Studien, die die institutionellen Auswirkungen auf die Repräsentation von Frauen im Parlament untersucht haben, haben internationale Vergleiche durchgeführt. In diesen Studien ist häufig die Repräsentationsrate von Frauen im Parlament die abhängige Variable (Kenworthy und Malami 1999; Moser 2001; Paxton et al. 2010; Reynolds 1999; Studlar und Mc Allister 2002; Valdini 2013). Trotz der bedeutenden Erkenntnisse und Analysemöglichkeiten, die Studien dieser Art bieten, gibt es auch Nachteile. Dadurch, dass die Analysen ausschließlich die Gewinner*innen fokussieren, ist es nicht möglich, auf individueller Ebene Faktoren zu identifizieren, die im Auswahlprozess diskriminierende Wirkung hatten. Individuelle Analysen machen es möglich, sozialstrukturelle Kontrollfaktoren (wie z. B. Alter oder Bildung) und institutionelle Faktoren (z. B. Wahlsystem, Parteienzugehörigkeit, Qualität der Nominierung) zu modellieren.

8 In Tab. 4 im Anhang sind die verwendeten Variablen und ihre deskriptiven Statistiken aufgeführt.

9 Das Alter wurde z-transformiert. 
Die zentrale erklärende Variable in allen Modellen ist das Geschlecht der Kandidat*innen (1: ,weiblich“, 0: „männlich“). Die Kontrollvariablen sind Alter", Bildung ${ }^{10}$ und - in Modellen des Wahlerfolgs - die Nominierungsqualität.

Weitere erklärende Variablen sind:

- Kandidaturtyp (0: „Doppelkandidatur“; 1: „Listenkandidatur“; 2: „Wahlkreiskandidatur") und

- Parteiideologie (0: ,rechtes Parteispektrum“; 1: „linkes Parteispektrum“).

Wie die theoretischen Ausführungen gezeigt haben, ist der entscheidende Faktor für den Wahlerfolg, wie aussichtsreich eine Kandidatur ist, weshalb die Nominierungsqualität einerseits als Kontrollvariable und andererseits als abhängige Variable in den Analysen berücksichtigt wird. Methodisch wurde zur Berechnung der Nominierungsqualität auf weit verbreitete Analysestrategien zurückgegriffen. Zur Unterscheidung zwischen aussichtsreichen und weniger aussichtsreichen Wahlkreisen wurde die Methode von Schmitt und Wüst (2004) verwendet: Ein Wahlkreis wird als ,aussichtsreich“ eingestuft, wenn der Abstand zwischen dem Gewinner bzw. der Gewinnerin des Wahlkreises und dem zweitplatzierten mindestens zehn Prozentpunkte beträgt (kodiert als 1); jeder andere Wahlkreis ist „,nicht aussichtsreich“ (kodiert als 0$).{ }^{11}$

Zur Bestimmung der Qualität von aussichtsreichen und weniger aussichtsreichen Listenplätzen wurde die Methode von Manow und Nistor (2009, S. 612) verwendet. Hierbei wird die Erfolgswahrscheinlichkeit einer Listenplatzierung anhand der Häufigkeit berechnet, mit der ein bestimmter Listenplatz einer bestimmten Partei in einem bestimmten Bundesland in der Vergangenheit zu einem Parlamentsmandat geführt hat. ${ }^{12}$ Aus beiden Berechnungen der Nominierungsqualität wurde eine gemeinsame Variable erstellt, die Auskunft darüber gibt, ob eine Kandidatur - unabhängig von einer Wahlkreis- oder Listenkandidatur - ,aussichtsreich“ (1) oder „nicht aussichtsreich“ (0) ist. ${ }^{13}$

\footnotetext{
${ }^{10}$ Mangels besserer Alternativen wurde der akademische Titel des Kandidierenden als Bildungsvariable verwendet - Doktorgrad oder Professorentitel (mit 1 kodiert) vs. kein Doktorgrad oder Professorentitel (mit 0 kodiert).

${ }^{11}$ Wahlkreise mit Veränderungen des Wahlkreiszuschnitts wurden mit 0 kodiert.

12 Auf der Grundlage aller vorangegangenen Bundestagswahlen wurde die Wahrscheinlichkeit berechnet, wann eine bestimmte Listenposition den Sitz bei der nächsten Wahl gewinnen wird. Manow und Nistor (2009) nennen dies die „Erfolgswahrscheinlichkeit“ und berechnen sie mit der folgenden Formel: $P_{\text {Position } i}=\frac{F_{\text {Rank } i}}{N_{w}}(P$ : Wahrscheinlichkeit, dass eine Listenposition in einer bestimmten Partei in einem bestimmten Staat das Mandat gewinnt (0-1); $i$ : Position auf der Parteiliste einer Partei in einem Bundesland; $F_{\text {Rank } i}$ : absolute Häufigkeit der Mandate, die diese Listenposition im Deutschen Bundestag über alle Legislaturperioden erreicht hat, $N_{w}$ : Gesamtzahl der enthaltenen Wahlen). Auf der Grundlage dieser Formel wurde die Erfolgswahrscheinlichkeit für jede Listenposition in jedem Jahr, für jede Partei in jedem Bundesland berechnet. Die berechneten Wahrscheinlichkeiten liegen zwischen 0 und 1, wobei eine Wahrscheinlichkeit von 1,0 eine Erfolgschance von 100\% für den/die Kandidat*in darstellt, während eine Wahrscheinlichkeit von 0,0 eine Erfolgschance von $0 \%$ bedeutet. Sowohl für die Berechnung der Qualität der Wahlkreise als auch der Listenplatzierungen wurde die vorherige Wahl bzw. die vorherigen Wahlen als Berechnungsgrundlage verwendet, weshalb die Analysen 1957 beginnen.

${ }^{13}$ Hierzu wurden Kandidat*innen mit einer Listenqualität von 0,7 und größer als aussichtsreich 1 kodiert. Ebenso wurden die nach der Methode von Schmitt und Wüst (2004) ermittelten aussichtsreichen Wahlkreise mit 1 kodiert. Die restlichen Kandidat*innen wurden mit 0 kodiert.
} 
Die Berechnungen erfolgen jeweils in Längsschnittmodellen von 1953-2017 und in Querschnittsmodellen, die nur die Kandidat*innen der Bundestagswahl 2017 umfassen. Die Längsschnittmodelle wurden nach Wahljahr geclustert.

\subsection{Ergebnisse}

Die zentrale Hypothese dieser Arbeit ist, dass Frauen bei Bundestagswahlen geringere Wahlchancen haben als Männer. Um diese Hypothese zu untersuchen, wurde ein Basismodell spezifiziert, das allein das Geschlecht als unabhängige Variable berücksichtigt. Ein weiteres, umfassendes Modell zieht alle erklärenden Variablen (Nominierungsqualität, Kandidatentyp, Parteiideologie) und Kontrollvariablen (Alter, Bildung) mit ein (Tab. 1).

Kann ein signifikant negativer Effekt der Variable „Geschlecht“ beobachtet werden? Im longitudinalen Basismodell (Model 1) zeigt sich zwar ein solch negativer Geschlechtseffekt, im Gesamtmodell ist die Variable „Geschlecht“ jedoch weder im Längsschnittmodell (Modell 3) noch im Modell 2017 (Modell 4) signifikant. Dies spricht zunächst gegen Hypothese 1 und somit gegen geringere Wahlchancen von Frauen bei Bundestagswahlen, sowohl im Längsschnitt als auch im Querschnitt 2017. Bieber (2013) fand jedoch heraus, dass es Unterschiede zwischen den Erfolgswahrscheinlichkeiten der Wahlsysteme gibt.

Tab. 1 Wahlerfolg

\begin{tabular}{|c|c|c|c|c|}
\hline & $\begin{array}{l}\text { Basis } \\
1953-2017 \\
(1)\end{array}$ & $\begin{array}{l}2017 \\
(2)\end{array}$ & $\begin{array}{l}\text { Gesamt } \\
\text { 1953-2017 } \\
\text { (3) }\end{array}$ & $\begin{array}{l}2017 \\
(4)\end{array}$ \\
\hline Frauen & $\begin{array}{l}-0,27 * * * \\
(0,08)\end{array}$ & $\begin{array}{c}-0,12 \\
(0,10)\end{array}$ & $\begin{array}{c}-0,00 \\
(0,08)\end{array}$ & $\begin{array}{c}-0,07 \\
(0,16)\end{array}$ \\
\hline Alter (standardisiert) & - & - & $\begin{array}{l}0,29 * * * \\
(0,04)\end{array}$ & $\begin{array}{l}0,15^{*} \\
(0,06)\end{array}$ \\
\hline Bildung (Titel) & - & - & $\begin{array}{l}0,41 * * * \\
(0,08)\end{array}$ & $\begin{array}{l}0,33 \\
(0,19)\end{array}$ \\
\hline Nominierungsqualität & - & - & $\begin{array}{l}4,01 * * * \\
(0,20)\end{array}$ & $\begin{array}{l}3,22 * * * \\
(0,15)\end{array}$ \\
\hline \multicolumn{5}{|c|}{ Kandidaturtyp (Referenz: Doppelkandidatur) } \\
\hline Nur Wahlkreis & - & - & $\begin{array}{l}-0,57 * \\
(0,26)\end{array}$ & $\begin{array}{l}-1,65 * * * \\
(0,18)\end{array}$ \\
\hline Nur Parteiliste & - & - & $\begin{array}{l}-2,52 * * * \\
(0,13)\end{array}$ & $\begin{array}{l}-3,85 * * * \\
(0,46)\end{array}$ \\
\hline Linke Parteien & - & - & $\begin{array}{l}-0,17 \\
(0,14)\end{array}$ & $\begin{array}{l}-0,57 * * * \\
(0,14)\end{array}$ \\
\hline Konstante & $\begin{array}{l}-0,73 \text { *** } \\
(0,05)\end{array}$ & $\begin{array}{l}-0,88 * * * \\
(0,05)\end{array}$ & $\begin{array}{l}-1,08 * * * \\
(0,10)\end{array}$ & $\begin{array}{l}-0,86^{* * * *} \\
(0,11)\end{array}$ \\
\hline$N$ & 30711 & 2414 & 30.711 & 2414 \\
\hline Pseudo $R^{2}$ & 0,002 & 0,000 & 0,508 & 0,465 \\
\hline
\end{tabular}

Einträge sind Logits-Koeffizienten und Standardfehler in Klammern. $* p<0,05 ; * * p<0,01 ; * * * p<0,001$ 
Tab. 2 Wahlerfolg, Interaktionseffekte

\begin{tabular}{|c|c|c|}
\hline & $\begin{array}{l}\text { Gesamt } \\
1953-2017 \\
(5)\end{array}$ & $\begin{array}{l}2017 \\
(6)\end{array}$ \\
\hline Frauen & $\begin{array}{l}0,02 \\
(0,12)\end{array}$ & $\begin{array}{l}-0,61 * \\
(0,26)\end{array}$ \\
\hline Alter (standardisiert) & $\begin{array}{l}0,29 * * * \\
(0,04)\end{array}$ & $\begin{array}{l}0,14 * \\
(0,06)\end{array}$ \\
\hline Bildung (Titel) & $\begin{array}{l}0,41 * * * \\
(0,08)\end{array}$ & $\begin{array}{l}0,32 \\
(0,19)\end{array}$ \\
\hline Nominierungsqualität & $\begin{array}{l}4,00 * * * \\
(0,20)\end{array}$ & $\begin{array}{l}3,23 * * * \\
(0,16)\end{array}$ \\
\hline \multicolumn{3}{|c|}{ Kandidaturtyp (Referenz: Doppelkandidatur) } \\
\hline Nur Wahlkreis & $\begin{array}{c}-0,40 \\
(0,26)\end{array}$ & $\begin{array}{l}-1,63 \text { *** } \\
(0,20)\end{array}$ \\
\hline Nur Parteiliste & $\begin{array}{l}-2,50^{* * * *} \\
(0,15)\end{array}$ & $\begin{array}{l}-3,78 * * * \\
(0,59)\end{array}$ \\
\hline Linke Parteien & $\begin{array}{c}-0,22 \\
(0,14)\end{array}$ & $\begin{array}{l}-0,81 * * * \\
(0,16)\end{array}$ \\
\hline \multicolumn{3}{|c|}{ Interaktionseffekte: Geschlecht * } \\
\hline Nur Wahlkreis & $\begin{array}{l}-1,95 * * * \\
(0,28)\end{array}$ & $\begin{array}{l}-0,32 \\
(0,59)\end{array}$ \\
\hline Nur Parteiliste & $\begin{array}{l}-0,06 \\
(0,12)\end{array}$ & $\begin{array}{l}-0,00 \\
(0,94)\end{array}$ \\
\hline Linke Parteien & $\begin{array}{l}0,29 \\
(0,16)\end{array}$ & $\begin{array}{l}0,96 * * \\
(0,32)\end{array}$ \\
\hline Konstante & $\begin{array}{l}-1,10^{* * * *} \\
(0,10)\end{array}$ & $\begin{array}{l}-0,78 * * * \\
(0,11)\end{array}$ \\
\hline$N$ & 30.711 & 2414 \\
\hline Pseudo $R^{2}$ & 0,511 & 0,468 \\
\hline
\end{tabular}

Einträge sind Logits-Koeffizienten und Standardfehler in Klammern.

$* p<0,05 ; * * p<0,01 ; * * * p<0,001$

Um auf wahlsystematische Effekte zu testen, wurden im Gegensatz zu Bieber (2013) geschlechtsspezifische Interaktionseffekte mit der Art der Kandidatur berechnet (Tab. 2). ${ }^{14}$ Die vorhergesagten Wahrscheinlichkeiten sind in Abb. 6 dargestellt. Der geschlechtsspezifische Interaktionseffekt mit reinen Wahlkreiskandidaturen zeigte im Längsschnittmodell (Modell 5) einen signifikanten negativen Geschlechtereffekt, der in Abb. 6a dargestellt ist. Demnach waren die Chancen von Frauen, die nur für den Wahlkreis kandidierten, signifikant schlechter als die von Männern, die nur für den Wahlkreis kandidierten. Geringere geschlechtsspezifische Unterschiede sind bei Doppelkandidaturen und reinen Listenkandidaturen festzustellen, wobei die Wahlchancen von Frauen mit Doppelkandidaturen im Längsschnitt besser sind als die von Männern. Kandidat*innen, die nur auf Listen kandidieren, haben, unabhängig vom Geschlecht, sehr geringe Chancen auf Erfolg. Hypothese 2

14 Bieber (2013) berechnete jeweils getrennte Modelle für einerseits Kandidaturen im Wahlkreis und andererseits Kandidaturen auf Landesliste. 
a

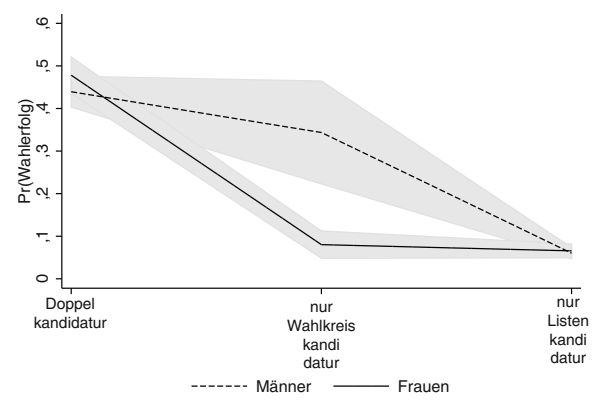

C

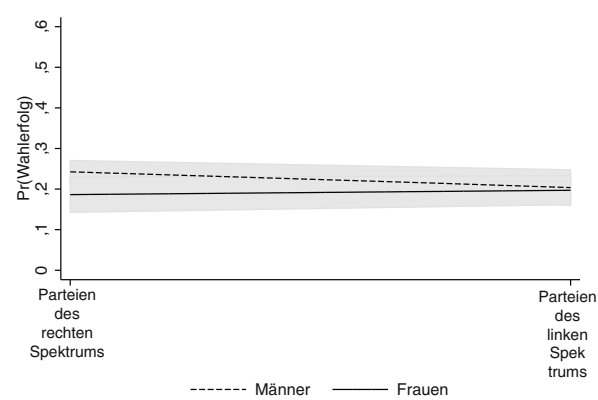

b
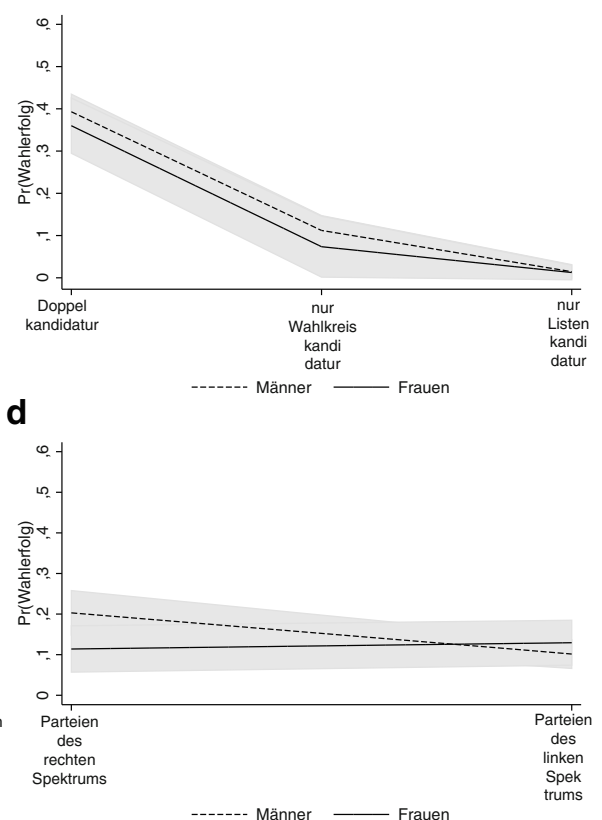

Abb. 6 Wahlerfolg, Wahlwahrscheinlichkeit. a Kandidaturtyp - 1953-2017, b Kandidaturtyp - 2017, c Parteispektrum - 1953-2017, d Parteispektrum - 2017

kann somit nur insofern gestützt werden, dass Frauen bei reinen Wahlkreiskandidaturen geringere Wahlchancen haben als bei Doppelkandidaturen. Bei Doppelkandidaturen fallen die Wahlchancen von Frauen im Längsschnitt verhältnismäßig positiv aus.

In Hypothese 3 wurde angenommen, dass die Wahlchancen von Frauen in Parteien des linken Spektrums höher sind als in Parteien des rechten Spektrums. Die geschlechtsspezifischen Interaktionseffekte in Tab. 2 stützen diese Hypothese für die Bundestagswahl 2017 (Modell 6), jedoch nicht für die Langzeitbetrachtung von 1953-2017 (Model 5). Abb. 6d zeigt, dass die Geschlechterunterschiede 2017 insbesondere aus den unterschiedlichen Wahlchancen der Männer resultieren: Diese haben in Parteien des rechten Spektrums deutlich höhere Chancen als Frauen. Ebenso haben Männer in rechten Parteien deutlich bessere Chancen als Männer in Parteien des linken Spektrums.

Schließlich zu Hypothese 4: Die Qualität der Nominierung von Frauen ist schlechter als die von Männern. Dies wurde mithilfe der Variable „Nominierungsqualität““ operationalisiert, die die Qualität der Wahlkreise und Listenplätze auf der Grundlage früherer Wahlen berechnet (Details siehe Abschn. 3.2). Zur Untersuchung der Hypothese wurden zunächst logistische Regressionen mit der abhängigen Variable „Nominierungsqualität“ (kodiert als Dummy-Variable) und den dargestellten Kontrollvariablen berechnet (Tab. 3, Modelle 7, 8). In Bezug auf die Gesamtqualität zeigt Modell 7, dass in der Längsschnittperspektive keine Geschlechterunterschiede 
Tab. 3 Nominierungsqualität

\begin{tabular}{|c|c|c|c|c|c|c|}
\hline & \multicolumn{2}{|l|}{ Gesamt } & \multicolumn{2}{|l|}{ Parteiliste } & \multicolumn{2}{|l|}{ Wahlkreis } \\
\hline & $\begin{array}{l}1953-2017 \\
\text { (7) }\end{array}$ & $\begin{array}{l}2017 \\
(8)\end{array}$ & $\begin{array}{l}\text { 1953-2017 } \\
\text { (9) }\end{array}$ & $\begin{array}{l}2017 \\
(10)\end{array}$ & $\begin{array}{l}1953-2017 \\
(11)\end{array}$ & $\begin{array}{l}2017 \\
(12)\end{array}$ \\
\hline Frauen & $\begin{array}{l}-0,00 \\
(0,08)\end{array}$ & $\begin{array}{l}0,49 * * * \\
(0,12)\end{array}$ & $\begin{array}{l}0,03 * * \\
(0,01)\end{array}$ & $\begin{array}{l}0,07 * * * \\
(0,02)\end{array}$ & $\begin{array}{l}-0,59 * * * \\
(0,15)\end{array}$ & $\begin{array}{l}-0,16 \\
(0,21)\end{array}$ \\
\hline $\begin{array}{l}\text { Alter (standar- } \\
\text { disiert) }\end{array}$ & $\begin{array}{l}0,49 * * * \\
(0,05)\end{array}$ & $\begin{array}{l}0,24 * * * \\
(0,05)\end{array}$ & $\begin{array}{l}0,06 * * * \\
(0,00)\end{array}$ & $\begin{array}{l}0,04 * * * \\
(0,01)\end{array}$ & $\begin{array}{l}0,36 * * * \\
(0,06)\end{array}$ & $\begin{array}{l}0,11 \\
(0,07)\end{array}$ \\
\hline Bildung (Titel) & $\begin{array}{l}0,41 * * * \\
(0,07)\end{array}$ & $\begin{array}{l}0,40 * * \\
(0,15)\end{array}$ & $\begin{array}{l}0,09 * * * \\
(0,01)\end{array}$ & $\begin{array}{l}0,08 * * * \\
(0,02)\end{array}$ & $\begin{array}{l}0,16 \\
(0,09)\end{array}$ & $\begin{array}{l}0,16 \\
(0,21)\end{array}$ \\
\hline Linke Parteien & - & - & $\begin{array}{l}0,35 * * * \\
(0,01)\end{array}$ & $\begin{array}{l}0,36 * * * \\
(0,02)\end{array}$ & $\begin{array}{l}-0,94 * * * \\
(0,19)\end{array}$ & $\begin{array}{l}-0,15 \\
(0,17)\end{array}$ \\
\hline \multicolumn{7}{|c|}{ Kandidaturtyp (Referenz: Doppelkandidatur) } \\
\hline Nur Wahlkreis & $\begin{array}{l}-1,12^{* * * *} \\
(0,18)\end{array}$ & $\begin{array}{l}-1,34 * * * \\
(0,15)\end{array}$ & - & - & - & - \\
\hline Nur Parteiliste & $\begin{array}{l}-2,88^{* * * *} \\
(0,22)\end{array}$ & $\begin{array}{l}-4,84^{* * * *} \\
(0,58)\end{array}$ & - & - & - & - \\
\hline Doppelkandidatur & $\begin{array}{l}-0,07 \\
(0,13)\end{array}$ & $\begin{array}{l}-0,54 * * * \\
(0,11)\end{array}$ & $\begin{array}{l}0,03 * \\
(0,01)\end{array}$ & $\begin{array}{l}-0,01 \\
(0,02)\end{array}$ & $\begin{array}{l}-0,88 * * \\
(0,28)\end{array}$ & $\begin{array}{l}-2,47 \text { *** } \\
(0,26)\end{array}$ \\
\hline Konstante & $\begin{array}{l}-0,54 * * * \\
(0,04)\end{array}$ & $\begin{array}{l}-0,61 * * * \\
(0,09)\end{array}$ & $\begin{array}{l}0,03 * * * \\
(0,00)\end{array}$ & $\begin{array}{l}-0,01 \\
(0,02)\end{array}$ & $\begin{array}{l}-1,18^{* * * *} \\
(0,20)\end{array}$ & $\begin{array}{l}-1,29 * * * \\
(0,15)\end{array}$ \\
\hline$N$ & 30.711 & 2414 & 24.970 & 1778 & 19.263 & 1779 \\
\hline Pseudo $R^{2} / R^{2}$ & 0,206 & 0,199 & 0,2668 & 0,2634 & 0,082 & 0,144 \\
\hline
\end{tabular}

Einträge sind in Modell 7, 8, 11 und 12 Logits-Koeffizienten und in Modell 9 und 10 Regressionskoeffizienten und Standardfehler in Klammern.

$* p<0,05 ; * * p<0,01 ; * * * p<0,001$

zu beobachten sind. Für 2017 lässt sich jedoch feststellen, dass die Qualität der Nominierungen bei Frauen signifikant besser ist als bei Männern.

Die deskriptiven Ergebnisse zeigten jedoch, dass signifikante Unterschiede in der Nominierungsqualität zwischen Wahlkreis- und Listenkandidaturen bestehen. Die getrennte Modellierung der Nominierungsqualität von Parteilisten (Modelle 9, 10) und von Wahlkreisen (Model 11, 12) geben hierüber Auskunft. Da die Nominierungsqualität der Listenkandidaturen als kontinuierliche Variable vorliegt, wurden lineare Regressionen in Modell 9 und 10 durchgeführt. Für die Berechnung der Nominierungsqualität in den Wahlkreisen wurden wiederum logistische Regressionen herangezogen. Modelle 9 und 10 in Tab. 3 zeigen signifikant positive Geschlechtereffekte auf der Ebene der Parteilisten sowohl im Längsschnitt als auch im Jahr 2017. Demnach wurden Frauen bei der Aufstellung von Parteilisten besser platziert als Männer. Das Gegenteil gilt für die Wahlkreise (Modelle 11, 12). Die multivariaten Ergebnisse bestätigen hier die Erkenntnisse der deskriptiven Analyse und zeigen, dass Frauen in der Längsschnittperspektive eine signifikant schlechtere Nominierungsqualität aufweisen als Männer. Bei der Wahl 2017 ist dieser Effekt jedoch nicht mehr signifikant. 


\section{Zusammenfassung und Ausblick}

Noch immer sind Frauen im Deutschen Bundestag unterrepräsentiert - und das, obwohl Parlamentarierinnen und Parlamentarier institutionalisierte Maßnahmen etablieren könnten, um die Repräsentanz von Frauen in der Politik zu erhöhen. Hierhin bestünde die Chance, dem im Grundgesetz festgeschriebenen Grundrecht der Gleichstellung von Frauen und Männern gerecht zu werden und zumindest in den eigenen Reihen zu verwirklichen. Doch können institutionelle Reformen den Frauenanteil im Bundestag überhaupt erhöhen, und wenn ja, wie? - Zur Beantwortung dieser beiden Fragen geben die Ergebnisse dieser Untersuchung wichtige Hinweise.

Deskriptiv wurde zunächst festgestellt, dass der Anteil der Frauen, die eine Wahlkreiskandidatur erringen, deutlich geringer ist als der Anteil an Frauen, die ein Listenmandat haben. Unter kontrollierten Bedingungen wurde jedoch auch festgestellt, dass Frauen insgesamt keine geringeren Wahlwahrscheinlichkeiten haben als Männer, wenn Listen- und Wahlkreiskandidaturen zusammen betrachtet werden. Dies gilt sowohl 2017 als auch im Längsschnitt. Somit ist Hypothese 1 - dass Frauen geringere Wahlchancen haben als Männer - insgesamt zu verwerfen. Es wird jedoch darauf hingewiesen, dass lediglich der Auswahlprozess von der Kandidatur zum Mandat beobachtet wurde und vorherig stattfindende Selektionsmechanismen keine Berücksichtigung fanden.

Zudem ist zu berücksichtigen, dass die Wahlchancen insgesamt im Fokus standen und nicht zwischen den Wahlchancen der Wahlkreiskandidat*innen und denen der Listenkandidat*innen unterschieden wurde. Um diesen Faktor zu berücksichtigen, wurde die Art der Kandidatur berücksichtigt, d.h. ob eine Person ausschließlich als Listenkandidat*in, ausschließlich als Wahlkreiskandidat*in oder als Doppelkandidat*in antrat. Deskriptiv lässt sich feststellen, dass der Frauenanteil an reinen Wahlkreiskandidaturen niedrig und in aussichtsreichen Wahlkreisen besonders niedrig ausfällt. Multivariate Analysen zeigen zudem, dass die Wahlchancen von Frauen mit nur einer Wahlkreiskandidatur im Vergleich zu Männern longitudinal besonders gering sind. Hier wird deutlich, dass Frauen durch die mehrheitliche Komponente des Wahlsystems erhebliche Nachteile erfahren. Wenn Frauen nur eine Wahlkreisoder nur eine Listenkandidatur innehaben, haben sie sehr geringe Wahlchancen. Diese erhöht sich jedoch merklich, wenn Frauen mit einer Doppelkandidatur in den politischen Wettbewerb gehen.

Hinsichtlich des Einflusses der Parteiideologie wurde deskriptiv festgestellt, dass der Anteil an weiblichen Kandidaturen in Parteien des linken Spektrums höher ist als in Parteien des rechten Spektrums. Dieser Effekt wurde für 2017 auch in der multivariaten Analyse bestätigt. Die Wahlchancen für Frauen waren 2017 höher, wenn diese sich in Parteien des linken Spektrums aufstellen ließen.

Bezüglich der Nominierungsqualität zeigten die deskriptiven Ergebnisse schließlich, dass weibliche Wahlkreiskandidaturen und Frauen in Parteien des rechten Spektrums schlechtere Qualitäten aufweisen, während diese bei Listenkandidaturen und in Parteien des linken Spektrums seit Anfang der 1990er-Jahre besser sind. In der multivariaten Untersuchung wurden in dieser Hinsicht zunächst keine Unterschiede festgestellt. Eine differenzierte Betrachtung der Nominierungsqualität auf Parteilisten einerseits und in Wahlkreisen andererseits ergab jedoch geschlechtsspezifische 
Unterschiede: Während Frauen bessere Platzierungen auf den Parteilisten im Längsund Querschnitt erfahren, sind ihre Nominierungsqualitäten in den Wahlkreisen im Längsschnitt signifikant schlechter als die der Männer, was ein zentraler Grund für die schlechten Wahlchancen von Frauen in ausschließlichen Wahlkreiskandidaturen zu sein scheint. Ob und inwieweit dieser negative Geschlechtereffekt bei der Nominierungsqualität durch die Art der Nominierung in den Parteien oder durch die Auswahlmechanismen der Wähler*innen zu erklären ist, kann im Rahmen dieses Artikels nicht endgültig beantwortet werden. Der Einfluss der Wähler*innen scheint jedoch weniger erklärungskräftig zu sein, da in Deutschland die Wahl der Wahlkreiskandidat*innen sehr stark mit der favorisierten Parteiwahl zusammenhängt und bereits bei der Nominierung von Wahlkreiskandidat*innen mit hoher Wahrscheinlichkeit davon ausgegangen werden kann, dass der/die nominierte Kandidat*in auch gewählt wird (Kaack 1969; Reiser 2013).

Aussagekräftiger erscheint der Auswahlprozess in Parteien vor der Nominierung von Kandidatinnen und Kandidaten, bei dem das Geschlecht ein zentrales Auswahlkriterium zu sein scheint. Dies gilt sowohl im Hinblick auf eine überdurchschnittlich hohe Nominierung von Frauen auf aussichtsreiche Listenplätze als auch in weniger aussichtsreichen Wahlkreisen. Ein Resultat dessen könnte gerade sein, dass die Frauen, die sich im Nominierungsprozess - gleichgültig ob bei der Aufstellung von Wahlkreisen oder Parteilisten - durchgesetzt haben, besondere und möglicherweise auch höhere Qualifikationen aufweisen als ihre männlichen Konkurrenten. Träfe dies zu, wäre sogar zu erwarten, dass Frauen aufgrund dessen bessere Wahlchancen hätten. Diese vorgelagerten Prozesse bei der Nominierung der Kandidat*innen bedürfen weitere Analysen.

Abschließend stellt sich die Frage, was institutionell getan werden könnte, um den Anteil der Frauen im Bundestag zu erhöhen. Die Analysen haben gezeigt, dass sich das Mehrheitswahlrecht in den Wahlkreisen tendenziell eher negativ auf die Nominierungsqualitäten und Wahlchancen von Frauen auswirkt. Ein naheliegender, aber radikaler Schritt wäre es, auf das Mehrheitswahlrecht in den Wahlkreisen zu verzichten und die Abgeordneten ausschließlich nach dem Verhältniswahlrecht zu rekrutieren. Gemessen an der geringeren absoluten Zahl der weiblichen Nominierungen in den Wahlkreisen ist zu vermuten, dass diese Maßnahme zu einer Erhöhung des Frauenanteils im Bundestag führen würde. Dies ist freilich ein gewagter und politisch schwer durchsetzbarer Schritt, der jedoch in der Debatte um eine Wahlrechtsreform zumindest diskutiert werden sollte.

Hinzuweisen ist darauf, dass sich diese Studie nur mit einem von vielen verschiedenen Gruppenmerkmalen beschäftigt, die potenzielle Kandidat*innen - bewusst oder unbewusst - sowohl bei der Nominierung als auch bei der Auswahl in einem Wahlkreis diskriminieren könnten. Weitere Merkmale, die womöglich die Chance auf eine Nominierung im Wahlkreis verringern, sind Alter, Migrationshintergrund, Beruf oder Religionszugehörigkeit. Durch die Etablierung eines ausschließlichen Verhältniswahlrechts mit geschlossener Parteiliste könnte auch in Hinblick auf diese soziostrukturellen Merkmale potenzielle politische Diskriminierung abgebaut werden. Wie die Beispiele der FDP und AfD jedoch zeigen, bedeutet ein reines Verhältniswahlrecht nicht automatisch, dass sich der Frauenanteil in den Mandaten auch erhöht. Beides sind Parteien, die ihre Mandate - mit Ausnahme der AfD im Os- 
ten - weitgehend über die Parteiliste gewinnen und einen verhältnismäßig niedrigen Frauenanteil im Bundestag stellen. Es ist jedoch davon auszugehen, dass aufgrund der unterschiedlichen Nominierungs- und Wahlkampfsituation von Wahlkreiskandidat*innen im Vergleich zu Listenkandidat*innen die Wahlchancen von Frauen, wie international bereits festgestellt, auch in Deutschland durch die Verhältniswahl erhöht würden. Möchte man das Mehrheitswahlrecht nicht komplett abschaffen, so bestände eine weitere Möglichkeit darin, den Effekt dieser Komponente zu verringern, indem der Anteil der Wahlkreismandate an Bundestagsmandaten insgesamt dezimiert wird.

Die vorliegenden Analysen haben gezeigt, dass auch die jeweilige Parteiideologie einen Einfluss darauf hat, wie hoch die Chance von Frauen auf ein Mandat ist. Eine weitere Maßnahme zur Förderung von Frauen wäre daher die Einführung einer rechtsverbindlichen Geschlechterquotierung für alle Parteien (z. B. in Form von paritätischen Wahllisten) mit entsprechenden Sanktionen bei Nichteinhaltung. Denn problematisch bei der aktuellen Umsetzung von parteiinternen Geschlechterquoten ist nicht nur ihre geringe Verbindlichkeit, sondern die Möglichkeit jeder Partei, frei darüber zu entscheiden, ob sie grundsätzlich eine Quotierung möchte oder nicht. In Deutschland wurden solche rechtsverbindlichen Geschlechterquoten bisher nur auf Landesebene in Form von Paritätsgesetzen in Brandenburg und Thüringen eingeführt. Die Landesverfassungsgerichte haben die Gesetze jedoch für verfassungswidrig erklärt, da sie u.a. gegen die Organisationsfreiheit, die Vorschlagsfreiheit, die Chancengleichheit, die Volkssouveränität und die freie Selbstbestimmung der Parteien verstoßen würden (Verfassungsgericht Thüringen 2020; Verfassungsgericht Brandenburg 2020). Offen und ungelöst bleibt jedoch die Diskrepanz zwischen diesen Entscheidungen und Art. 3 Abs. 3 GG - dem Grundrecht der Gleichberechtigung von Frauen und Männern und der Verantwortung des Staates, diese herbeizuführen und dem Ziel der Wahlrechtsreform, eine ,gleichberechtigte Repräsentation von Frauen und Männern auf den Kandidatenlisten und im Bundestag zu erreichen.“ 


\section{Anhang}

Tab. 4 Variablen

\begin{tabular}{|c|c|c|c|c|}
\hline $\begin{array}{l}\text { Name der Varia- } \\
\text { blen }\end{array}$ & Kodierung & $N$ & Min & Max \\
\hline Frauen & $\begin{array}{l}\text { (0) Männer } \\
\text { (1) Frauen }\end{array}$ & 32.436 & 0 & 1 \\
\hline Alter in Jahren & $\begin{array}{l}\text { (18) } 18 \text { Jahre } \\
\text { (89) } 89 \text { Jahre }\end{array}$ & 32.434 & 18 & 89 \\
\hline Titel & $\begin{array}{l}\text { (0) kein Titel } \\
\text { (1) Dr. oder Professortitel }\end{array}$ & 32.436 & 0 & 1 \\
\hline Wahljahr & $\begin{array}{l}(1953) 1953 \\
\ldots \\
(2017) 2017\end{array}$ & 32.436 & 1953 & 2017 \\
\hline $\begin{array}{l}\text { Linkes Partei- } \\
\text { spektrum }\end{array}$ & $\begin{array}{l}\text { (0) rechtes Parteispektrum } \\
\text { (1) linkes Parteispektrum }\end{array}$ & 32.436 & 1 & 7 \\
\hline Kandidaturtyp & $\begin{array}{l}\text { (0) Doppelkandidatur } \\
\text { (1) Wahlkreiskandidatur } \\
\text { (2) Listenkandidatur }\end{array}$ & 32.436 & 0 & 2 \\
\hline $\begin{array}{l}\text { Wahlerfolg ge- } \\
\text { samt }\end{array}$ & $\begin{array}{l}\text { (0) nein } \\
\text { (1) ja }\end{array}$ & 32.436 & 0 & 1 \\
\hline $\begin{array}{l}\text { Nominierungsqu } \\
\text { gesamt }\end{array}$ & $\begin{array}{l}\text { (0) aussichtslose Listen- oder/und Wahlkreiskan- } \\
\text { didatur } \\
\text { (1) aussichtsreiche Listen- oder/und Wahlkreis- } \\
\text { kandidatur }\end{array}$ & 30.711 & 0 & 1 \\
\hline $\begin{array}{l}\text { Nominierungsqu } \\
\text { Wahlkreis }\end{array}$ & $\begin{array}{l}\text { (0) aussichtslose Wahlkreisnominierung } \\
\text { (1) aussichtsreiche Wahlkreisnominierung }\end{array}$ & 19.263 & 0 & 1 \\
\hline $\begin{array}{l}\text { Nominierungsqu } \\
\text { Parteiliste }\end{array}$ & $\begin{array}{l}\text { (0) aussichtslose Listennominierung } \\
\text {... } \\
\text { (1) aussichtsreiche Listennominierung }\end{array}$ & 24.970 & 0 & 1 \\
\hline
\end{tabular}


Förderung Diese Arbeit wurde vom Gleichstellungsbüro der Goethe-Universität Frankfurt am Main und dem Fachbereich 03/Goethe-Universität Frankfurt am Main unterstützt.

Conflict of Interest I. E. Bieber has no competing interests to declare that are relevant to the content of this article.

\section{Literatur}

Abels, Gabriele, Petra Ahrens, und Agnes Blome. 2018. Geschlechtergerechte Repräsentation in historischer und internationaler Perspektive. APuZ 42:28-36.

Allik, Mirjam. 2015. Who stands in the way of women? Open vs. closed lists and candidate gender in Estonia. East European Politics 31:429-451. https://doi.org/10.1080/21599165.2015.1084924.

Bernstein, Robert A. 1986. Why are there so few women in the house? The Western Political Quarterly 39:155-164. https://doi.org/10.2307/448421.

Bieber, Ina. 2013. Frauen in der Politik. Einflussfaktoren auf weibliche Kandidaturen zum Deutschen Bundestag. Wiesbaden: Springer VS.

Bieber, Ina. 2020. Luftloch oder Sturzflug? Sinkende Vertretung von Frauen in deutschen Parlamenten. Femina Politica - Zeitschrift für feministische Politikwissenschaft https://doi.org/10.3224/ feminapolitica.v29i1.08.

BMBF. 2020. 4. Atlas zur Gleichstellung von Frauen und Männern in Deutschland. https://www.bmfsfj. de/resource/blob/160308/73cf50519fdd0b72be8bce59a041079b/4--atlas-zur-gleichstellung-vonfrauen-und-maennern-in-deutschland-broschuere-data.pdf. Zugegriffen: 25. Apr. 2021.

Brunsbach, Sandra. 2011. Machen Frauen den Unterschied? Parlamentarierinnen als Repräsentantinnen frauenspezifischer Interessen im Deutschen Bundestag. Zeitschrift für Parlamentsfragen 42:3-24. https://doi.org/10.5771/0340-1758-2011-1-3.

Bundestag. 2020. Bundestag stimmt Koalitionsvorschlag zur Wahlrechtsänderung zu. https://www. bundestag.de/dokumente/textarchiv/2020/kw41-de-bundeswahlgesetz-796006. Zugegriffen: 25. Apr. 2021.

Bundestag. 2021. Grundgesetz für die Bundesrepublik Deutschland. https://www.bundestag.de/gg. Zugegriffen: 27. Apr. 2021.

Caul Kittilson, Miki. 2006. Challenging parties, changing parliaments. Women and elected office in contemporary Western Europe. Columbus: Ohio State Univ. Press.

Caul, Miki. 2001. Political parties and the adoption of candidate gender quotas. A cross-national analysis. The Journal of Politics 63:1214-1229. https://doi.org/10.1111/0022-3816.00107.

Davidson-Schmich, Louise. 2016. Gender quotas and democratic participation: recruiting candidates for elective offices in Germany. Michigan: University of Michigan Press.

die tageszeitung. 23. Nov. 2005. Deutschland auf Weltniveua. die tageszeitung (taz) 27: 1 .

Erzeel, Silvia, und Didier Caluwaerts. 2015. Is it gender, ideology or resources? Individual-level determinants of preferential voting for male or female candidates. Journal of Elections, Public Opinion and Parties 25:265-283. https://doi.org/10.1080/17457289.2015.1008495.

Fortin-Rittberger, Jessica, und Christina Eder. 2013. Towards a gender-equal Bundestag? The impact of electoral rules on women's representation. West European Politics 36:969-985. https://doi.org/10. 1080/01402382.2013.796702.

Hellmann, Daniel. 2020. Der mühselige Weg zum Mandat - aber welcher? Empirische Untersuchungen zu Inhalt und Bedeutung der Ochsentour. Zeitschrift für Parlamentsfragen 51:51-69.

Inglehart, Ronald, und Pippa Norris. 2003. Rising tide. Gender equality and cultural change around the world. Cambridge: Cambridge-University-Press.

Kaack, Heino. 1969. Wahlkreisgeographie und Kandidatenauslese: regionale Stimmenverteilung, Chancen der Kandidaten und Ausleseverfahren, dargestellt am Beispiel der Bundestagswahl 1965. Köln: Westdeutscher Verlag.

Kenworthy, L., und M. Malami. 1999. Gender inequality in political representation. A worldwide comparative analysis. Social Forces 78:235-268. https://doi.org/10.1093/sf/78.1.235.

Luhiste, Maarja. 2015. Party Gatekeepers' support for viable female candidacy in PR-list systems. Politics \& Gender 11:89-116. https://doi.org/10.1017/S1743923X14000580.

Manifesto Project. 2021. https://manifestoproject.wzb.eu/. Zugegriffen: 4. Sept. 2021.

Manow, Philip, und Martina Nistor. 2009. Wann ist ein Listenplatz sicher? Eine Untersuchung der Bundestagswahlen 1953 bis 2002. Zeitschrift für Parlamentsfragen 40:603-620. 
Matland, Richard, und Donley Studlar. 1996. The contagion of Wemen candidates in single-member district and proportional representation electoral systems: Canada and Norway. The Journal of Politics 58:707-733. https://doi.org/10.2307/296439.

Moser, Robert G. 2001. The effects of electoral systems on women's representation in post-communist states. Electoral Studies 20:353-369. https://doi.org/10.1016/S0261-3794(00)00024-X.

Norris, Pippa. 2004. Electoral engineering. Voting rules and political behavior. Cambridge: Cambridge University Press.

Ondercin, Heather L., und Susan Welch. 2009. Comparing predictors of women's congressional election success: candidates, primaries, and the general election. American Politics Research 37:593-613. https://doi.org/10.1177/2F1532673X08325198.

Paxton, Pamela, Melanie M. Huges, und Matthew A. Painer. 2010. Growth in women's political representation. A longitudinal exploration of democracy, electoral system and gender quotas. European Journal of Political Research 49:25-52. https://doi.org/10.1111/j.1475-6765.2009.01886.x.

Preece Robinson, Jessica, Olga Bogach Stoddard, und Rachel Fisher. 2016. Run, Jane, run! Gendered responses to political party recruitment. Political Behavior 38:561-577. https://doi.org/10.1007/ s11109-015-9327-3.

Reiser, Marion. 2013. Ausmaß und Formen des innerparteilichen Wettbewerbs auf der Wahlkreisebene. Nominierung der Direktkandidaten für die Bundestagswahl 2009. In Koalitionen, Kandidaten, Kommunikation. Analysen zur Bundestagswahl 2009, Hrsg. Thorsten Faas, Kai Arzheimer, Sigrid Roßteutscher, und Bernhard Weßels, 129-147. Wiesbaden: Springer.

Reynolds, Andrew. 1999. Women in the legislatures and executives of the world. Knocking at the highest glass ceiling. World Politics 51:547-572.

Rule, Wilma. 1987. Electoral systems, contextual factors and women's opportunity for election to parliament in twenty-three democracies. Political Research Quarterly 40:477-498. https://doi.org/10.1177/ 106591298704000307.

Scherer, Philipp. 2020. Links und rechts im Wandel. Zur Bedeutung und Relevanz der Richtungsbegriffe in Deutschland. Wiesbaden: Springer.

Schmitt, Hermann, und Andreas Wüst. 2004. Direktkandidaten bei der Bundestagwahl 2002: Politische Agenda und Links-Rechts-Selbsteinstufung im Vergleich zu den Wählern. In Die Bundestagswahl 2002: Analysen der Wahlergebnisse und des Wahlkampfes, Hrsg. Frank Brettschneider, Jan W. van Deth, und Edeltraud Roller, 303-325. Wiesbaden: VS.

Schwindt-Bayer, Leslie A. 2005. The incumbency disadvantage and women's election to legislative office. Electoral Studies 24:227-244. https://doi.org/10.1016/j.electstud.2004.05.001.

Siaroff, Alan. 2000. Women's representation in legislatures and cabinets in industrial democracies. International Political Science Review / Revue internationale De Science Politique 21:197-215.

Stockemer, Daniel, und Aksel Sundström. 2017. Women in cabinets: The role of party ideology and government turnover. Party Politics 24:663-673. https://doi.org/10.1177/1354068817689954.

Studlar, Donley T., und Ian Allister Mc. 2002. Does a critical mass exist? A comparative analysis of women's legislative representation since 1950. European Journal of Political Reseach 41:233-253. https://doi.org/10.1111/1475-6765.00011.

UN Women. 2021. Facts and figures: Womens's leadership and political participation. https://www. unwomen.org/en/what-we-do/leadership-and-political-participation/facts-and-figures. Zugegriffen: 23. Mai 2021.

Valdini, Melody Ellis. 2013. Electoral institutions and the manifestation of bias. The effect of the personal vote on the representation of women. Politics \& Gender 9:76-92. https://doi.org/10.1017/ S1743923X12000700.

Vengroff, Richard, Zsolt Nyiri, und Melissa Fugiero. 2003. Electoral system and gender representation in sub-national legislatures. Is there a national-sub-national gender gap? Political Research Quarterly 56:163. https://doi.org/10.2307/3219895.

Verfassungsgericht Brandenburg. 2020. Pressemitteilung: Paritätsgesetz verfassungswidrig. https:// verfassungsgericht.brandenburg.de/verfgbbg/de/presse-statistik/pressemitteilungen/detail/ 23-102020-paritaetsgesetz-verfassungswidrig. Zugegriffen: 27. Apr. 2021.

Verfassungsgericht Thüringen. 2020. Urteil. http://www.thverfgh.thueringen.de/webthfj/webthfj.nsf/ 8104B54FE2DCDADDC12585A600366BF3/\$File/20-00002-U-A.pdf?OpenElement. Zugegriffen: 27. Apr. 2021. 